\title{
Evaluation of TRMM Summer Precipitation Over Huai-River Basin In China
}

\author{
Man Zhang ${ }^{1}$, Chen Sheng ${ }^{2}$, Youcun Qi ${ }^{1 a}$, Yi Yang $^{3}$ \\ ${ }^{1}$ College of Atmospheric Sciences, Nanjing University of Information Science \& Technology, Nanjing \\ 210044, China \\ ${ }^{2}$ Hydrometeorology and Remote Sensing Laboratory, University of Oklahoma, Norman, OK, \\ 73072,USA \\ ${ }^{3}$ Key Laboratory for Semi-Arid Climate Change of the Ministry of Education, Key Laboratory of Arid \\ Climatic Changing and Reducing Disaster of Gansu Province, College of Atmospheric Sciences, \\ Lanzhou University, Lanzhou, 730000, China \\ aYoucun.Qi@gmail.com
}

\section{Keywords: Huaihe Basin, TRMM, Precipitation}

\begin{abstract}
In this study, the biases and uncertainty of TRMM 3B42 estimates are investigated over the Huai-River Basin during the summer season in 2010. TRMM products of 3B42RT, 3B42V6 and 3B42V7 are cross-compared to Chinese Precipitation Analyses Products (CPAP) as the reference. It is found that the distribution of bias is closely depend on the terrain with the dry bias locates at hills/mountains and wet bias lies on the plains area. It is concluded that the bias may be caused by the defect of TRMM algorithm which cannot discern different types of precipitation. 3B42V7 product shows the best improvement in reducing both wet and dry bias; it also appears small uncertainty on summer season precipitation estimate.
\end{abstract}

\section{Introduction}

Huai-river basin is the nationwide agriculture center, which is also the head of densely populated area. It plays very important role in sustaining the development and stableness over the whole country. Moreover, serious disasters like drought or flood often occur in this area, especially in the summer season. Therefore, the focus on weather variability becomes more and more important and is already involved in people's daily life. To better understand the mechanism of short scale weather phenomenon or improve the precision of numerical model forecast, the observation data of precipitation is absolutely important. Gauge observations provide relatively accurate data at the station locations, but the quality of data analysis closely depends on the density and the configuration of the gauge station networks (Morrissey et al. 1995). Limited by the representativeness of in-situ measurements from rain gauges and disdrometers, the challenge of precipitation measurement comes with complex variability of the precipitation itself in temporal and spatial dimensions (Gourley et al. 2010). The space- and ground- based remote-sensing instruments could provide high resolution precipitation data in temporal and spatial dimensions by adopting indirect way to measure the precipitation, but the set of instrument-specific errors and the defect of algorithm contribute to arising errors of the data. For this reason, accurate measurement and estimation of uncertainty of the data is essential for a variety of scientific topics (AMITAI et al. 2009; Hossain; Huffman 2008; Scofield; Kuligowski 2003).

In the last decades, the tropic precipitation monitoring project named TRMM (Tropic Rainfall Measurement Mission) has been well established for measuring tropical rainfall and investigating the physical process of atmospheric dynamics. The data obtained from TRMM is also very important for monitoring the global hydrological cycle, estimating the realism of climate model by comparing the strength and location of ITCZ (Equatorial Intertropical Convergence Zone) with those from model outputs. The set of TRMM products present good performance in improving the understand of tropic phenomenon as ENSO and ITCZ, but when they are applied to the extrotropic studies, the uncertainty and accuracy of this data should be discussed and resolved (Hirpa et al. 2010; Huffman et al. 2003; Huffman et al. 2001; Scofield; Kuligowski 2003). 
This work aims to finish the following goals: 1) describe the biases with spatial and temporal distributions of TRMM precipitation products relative to Chinese Precipitation Analyses Products (CPAP). 2) elucidate the uncertainty of TRMM products and provide the underlying causes of these issues which may contribute a valuable feedback to the algorithm developers in order to improve the accuracy of data.

\section{Data and Method}

\subsection{TRMM estimates}

The 3B42 estimates (3B42RT, 3B42V6, 3B42V7) are comprised of the TRMM real-time high quality (HQ) passive microwave precipitation and the TRMM real-time Variable Rain Rate (VAR) merged-infrared (IR) precipitation. Time interval is every 3-hour and spatial horizontal resolution is on a 0.25 degree by 0.25 degree grid, the estimates extend from $50 \mathrm{oN}$ to $50 \mathrm{oS}$. The algorithms which produce the estimates contribute to the difference between each product of 3B42. The detail information and the access of TRMM products can be found at http://daac.gsfc.nasa.gov/precipitation/TRMM_README/TRMM_3B42_readme.shtml.

\subsection{CAPA data}

Gauge data is considered to be accurate and stable since it is obtained by direct way to measure the precipitation. It also plays an important in evaluating the remote sensing data as the reference. Recently, by collecting hourly precipitation information from over 2000 automatic weather station over China, the National Meteorology Information Center (NMIC) of the China Meteorological Administration (CMA) developed an objective analysis system. With this system, an hourly Chinese Precipitation Analyses Products (CPAP) is created on a 0.25 degree by 0.25 degree grid. The data is available at http://cdc.cma.gov.cn/sksj.do?method=ssrjscp.Taking advantage of this gauge-based analysis, we will evaluate the performance of TRMM 3B42 products on summer precipitation estimation in 2010.

\subsection{Method}

In order to objectively evaluate the TRMM estimates, we use the following statistics methods: relative bias $(\mathrm{RB})$, root-mean-squared difference (RMSD), where

$$
\begin{gathered}
R B=\frac{\sum_{i=1}^{N} Y_{i}-\sum_{i=1}^{N} \operatorname{Re} f_{i}}{\sum_{i=1}^{N} \operatorname{Re} f_{i}} \times 100 \% \\
R M S D=\sqrt{\frac{1}{N} \sum_{i=1}^{N}\left(T_{i}-\operatorname{Re} f_{i}\right)^{2}}
\end{gathered}
$$

Where, i denotes the individual sample varying with time or spatial dimension, $\mathrm{N}$ represents for total number of spatio-temporal domain over which the statistic is computed (for instant, seasonal, annual, specific area). T denotes for TRMM estimates and Ref represents for CPAP data. When RB $>0$, it represents for wet bias, thus, $\mathrm{RB}<0$, it denotes for dry bias. When RMSD equals to zero, the product is perfect without any bias.

\section{Results}

Figures 1a-d show accumulated precipitation over summer season during the period from Jan 2010 to Dec 2010 estimated by CPAP and 3B42 TRMM products in this study. Each panel depicts similar precipitation patterns over Huai-river basin, the intensive precipitation locates at the area of $29^{\circ} \mathrm{N}-32^{\circ} \mathrm{N}, 114^{\circ}-118^{\circ} \mathrm{E}$. But there are notable differences of 3B42 TRMM products compared with the CPAP data set, precipitation of 3B42RT is overestimated at the southern and underestimated at the eastern. Other products of 3B42V6 and 3B42V7 do much better than 3B42RT in reducing the 
overestimation in the south $\left(28-32^{\circ} \mathrm{N}, 114-120^{\circ} \mathrm{E}\right)$ and underestimation in the middle west $\left(32-34^{\circ} \mathrm{N}, 107-111^{\circ} \mathrm{E}\right)$ of the panel , moreover, 3B $42 \mathrm{~V} 7$ have a great improvement in depicting the location of precipitation.
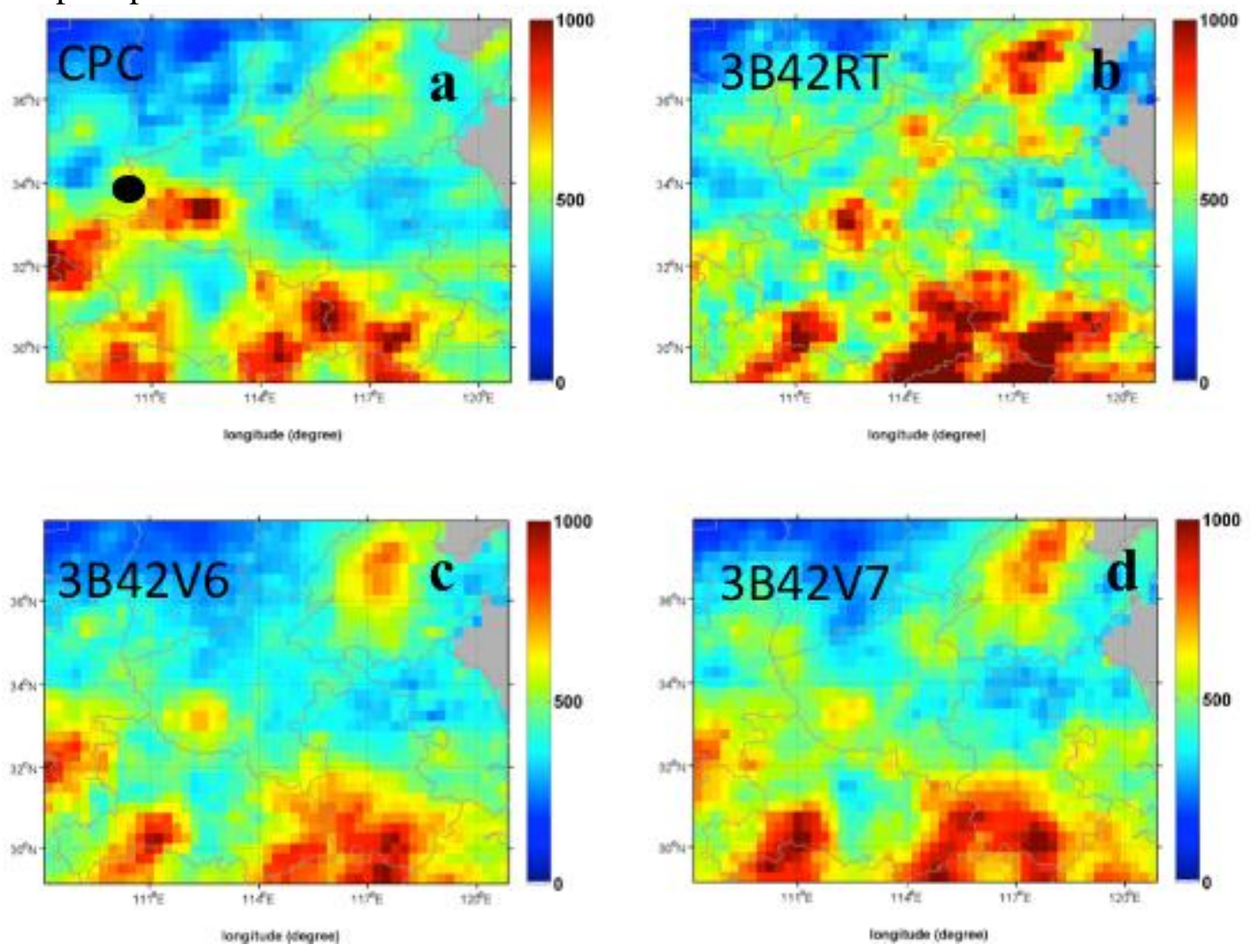

Fig.1 Accumulated summer precipitation bias (unit: mm) from a) 3B42RT, b) 3B42V6, c) 3B42V7. Red mark in a) represents for the origin of Huai-River. Black mark in a) represents for the origin of Huai-River.

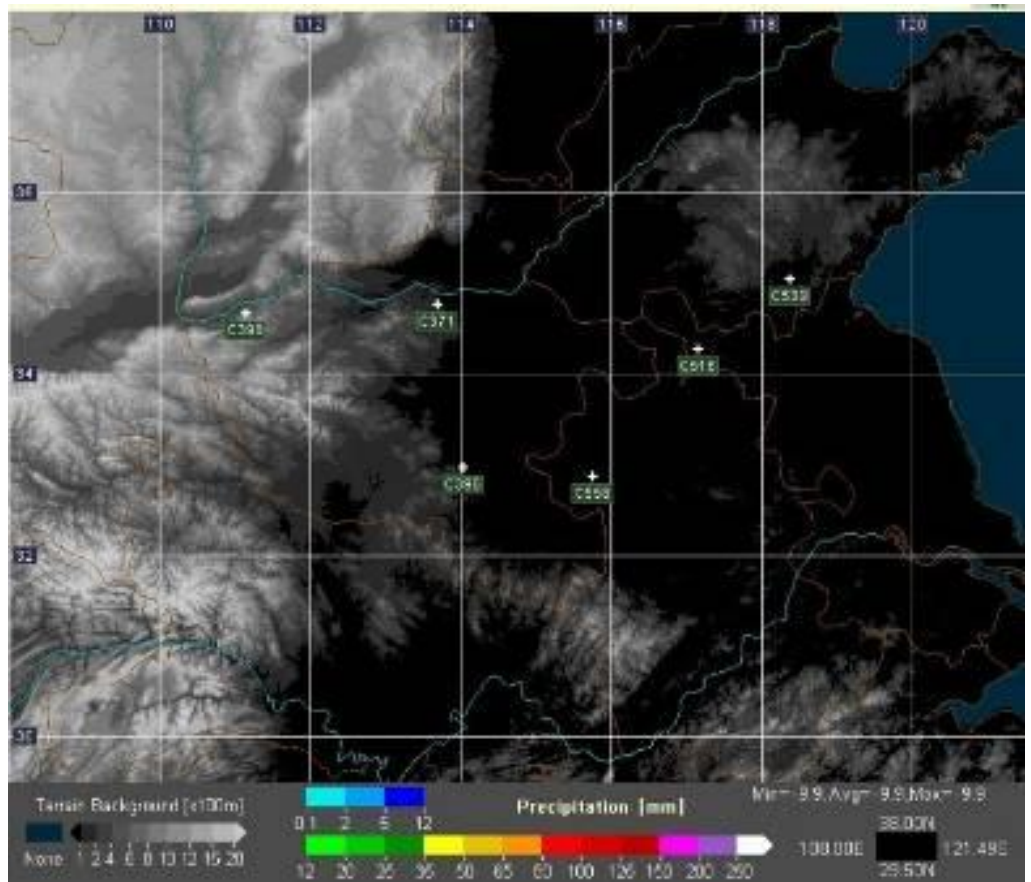

Fig. 2 Topography and Map of Huai-River basin. 

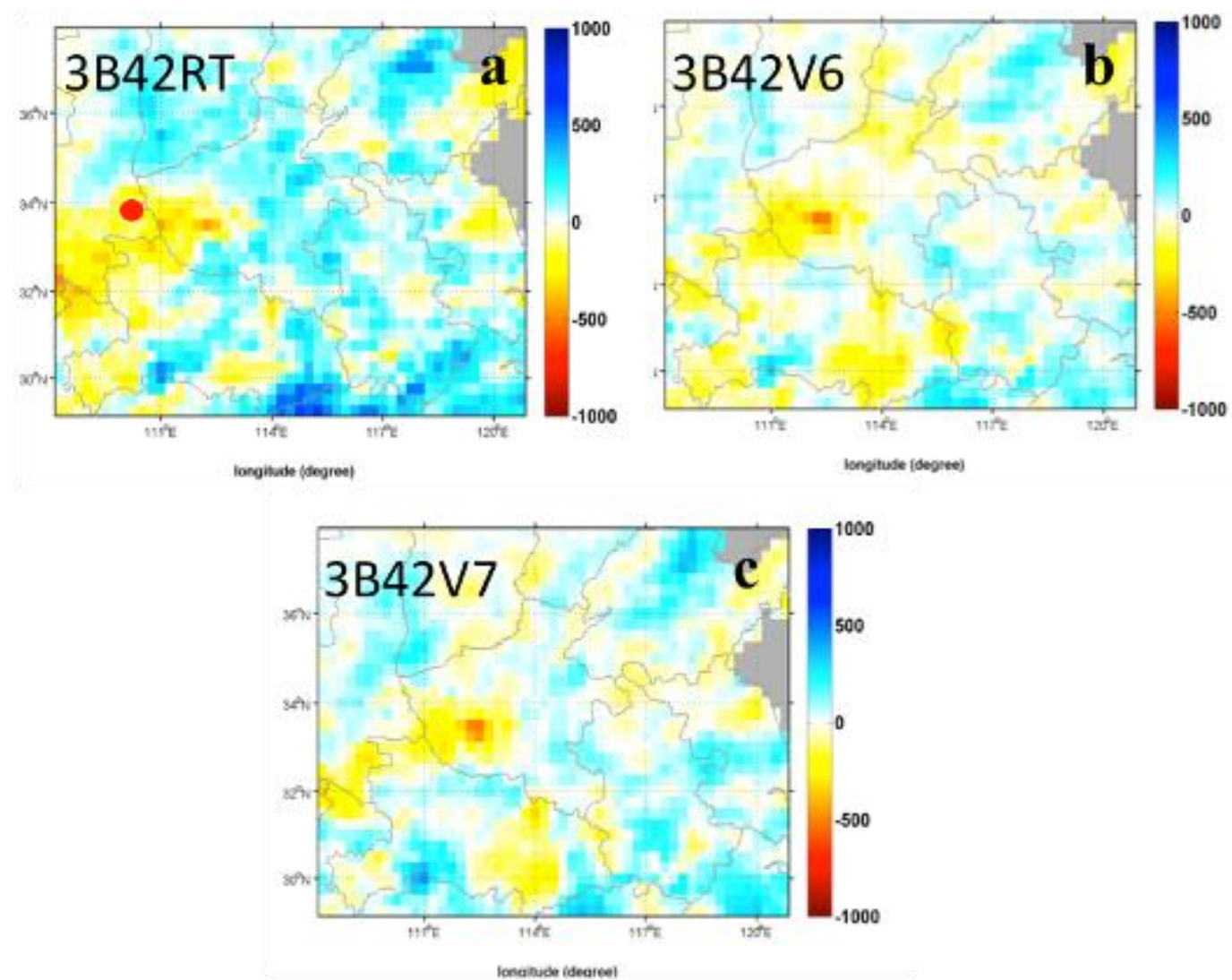

Fig.3 Accumulated summer precipitation bias (unit: mm) from a) 3B42RT, b) 3B42V6, c) 3B42V7

Fig. 3 shows the bias of accumulated precipitation during the summer season. By and large, each product depicts the similar bias pattern over Huai-river basin, notable dry bias in the west and wet bias in the East. Compared to topography distribution over the Huai-river basin (as shown in Fig.2), it is found to be interested that the dry bias locates at the hills and the wet bias lies on the plain. Therefore, we conclude that topography leads to different types of precipitation, at the meanwhile, the algorithm of TRMM estimates could not discern the differences of precipitation which located at between hills and plains. In this case, the defect of algorithm causes the systematic dry bias over the hills and wet bias over the plain. Further analysis shows that there are some visible differences among the estimates. Compared to 3B42RT, there are evident improvements of 3B42V6 and V7 products in decreasing both dry bias and wet bias. Furthermore, 3B42V6 does better in weakening the dry bias in the east, whereas, 3B42V7 removes most of the dry bias in the west and decreases the wet bias in the east. Overall, even though wet and dry biases still exit, 3B42V6 and 3B42V7 products have apparent improvement in removing biases of the precipitation.

Relative bias shows objectively estimations of 3B42 products, it is found that 3B42RT represents high dry bias at the eastern and wet bias at the western, 3B42V6 have good improvement in reducing the wet bias, but it appears a little dry bias of the wet bias. 3B42V7 does the best performance among the TRMM products in reducing both the dry and wet bias.

The RMSE pattern depicts the uncertainty of the TRMM estimations during the summer season. From Fig. 5a, it is shown that the uncertainty of 3B42RT product is very high over the most place of Huai-river basin, especially at the south boarder and the northeast part. 3B42V6 product could reduce the uncertainty of the summer precipitation in amplitude, but the uncertain area coverage is still large (shown in Fig.5c). 3B42V7 indicates an improved accuracy of summer precipitation in both weakening the amplitude of RMSE and reducing the uncertain area coverage. 

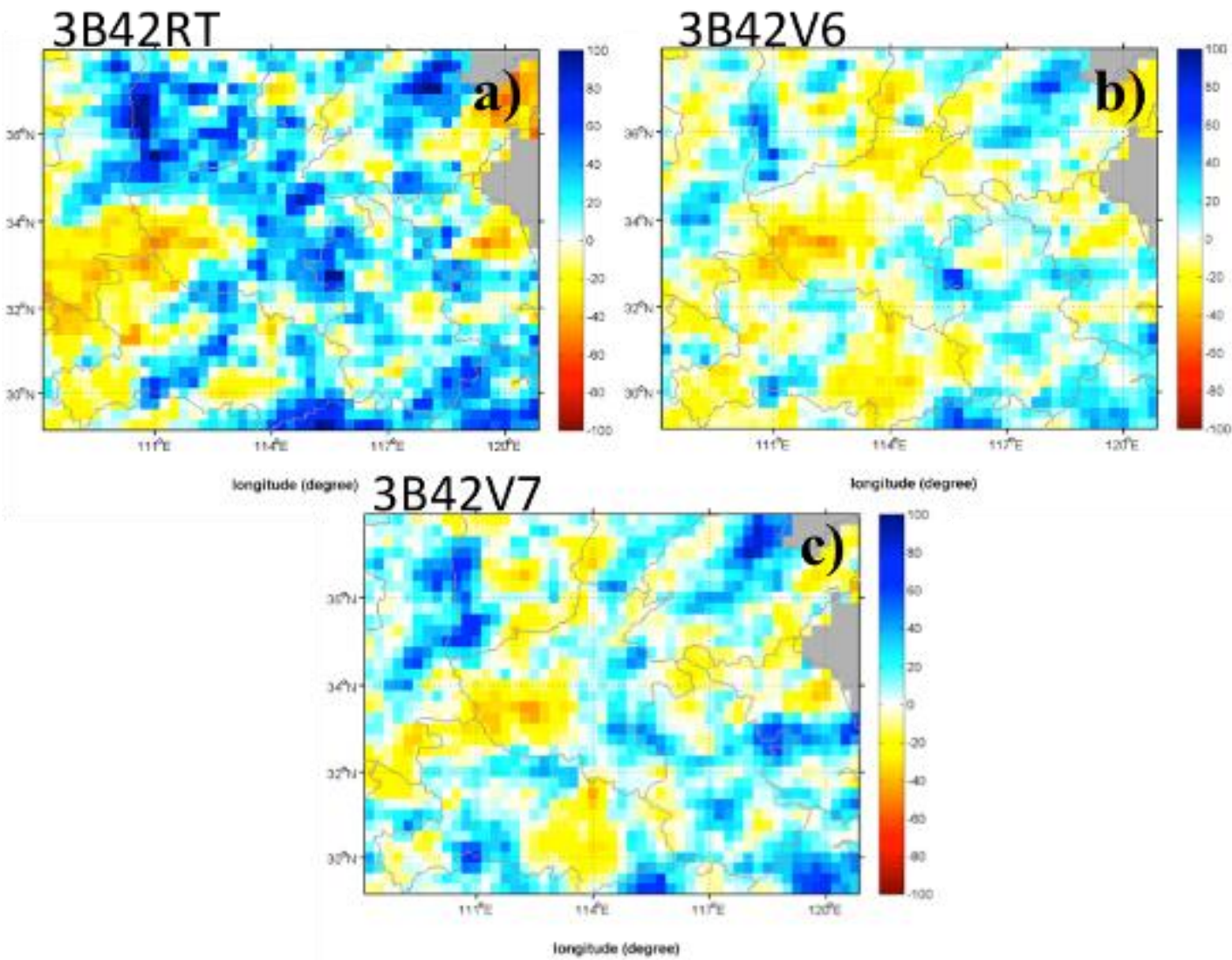

Fig.4 Same as Fig.3, but for relative bias
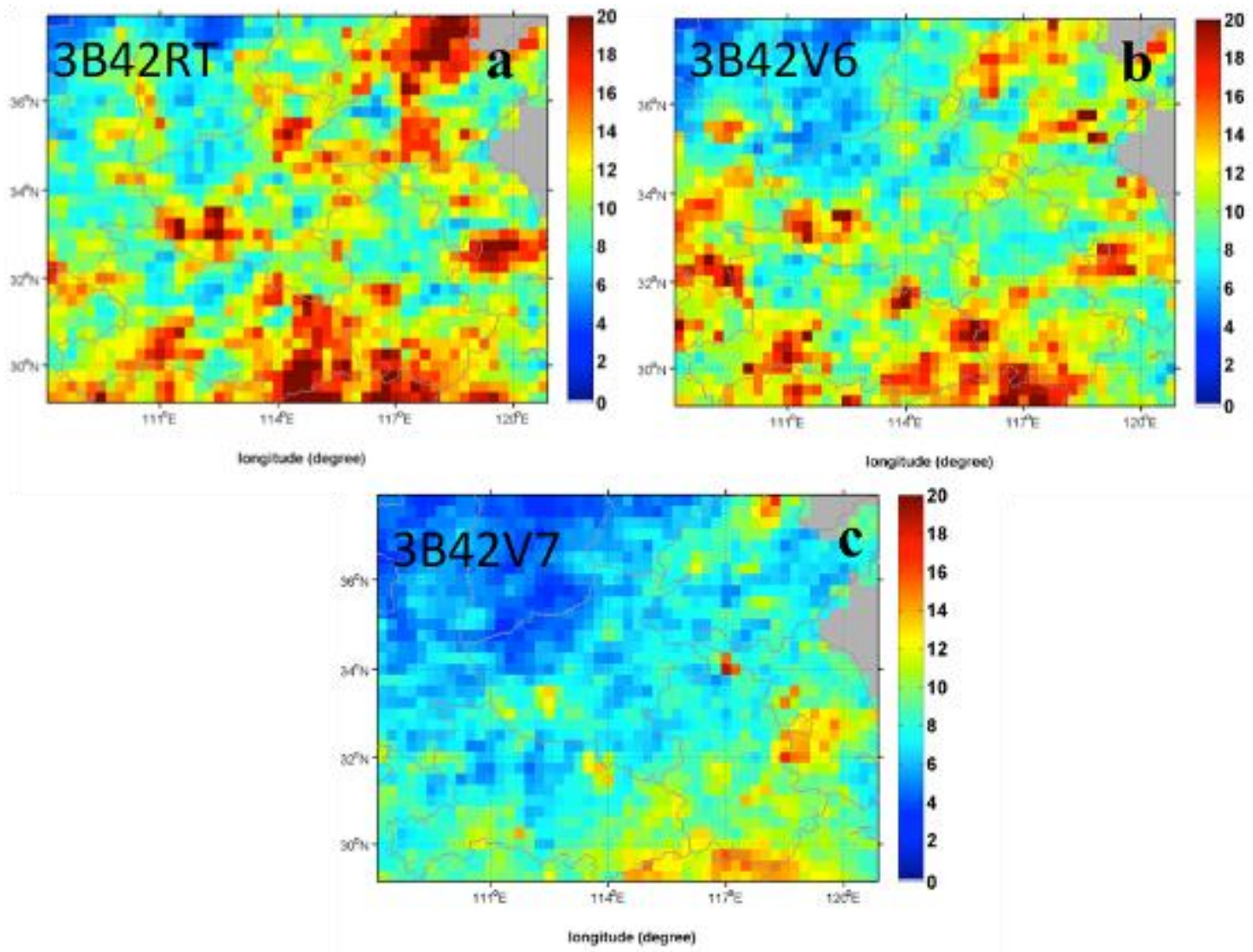

Fig.5 RMSE of summer precipitation with a) 3B42RT, b) 3B42V6, c) 3B42V7. 


\section{Conclusions}

TRMM 3B42 estimates provide high temporal and spatial resolution precipitation products by combining the microwave, infrared and radar data. From the statistic results of summer precipitation, it is found that dry bias mainly occurs at hills/mountains area and wet bias arises in plains. They might be caused by the defect of algorithms, which cannot discern different types of summer precipitation due to terrain. 3B42 $\mathrm{V} 7$ product shows a great improvement in reducing the bias and enhancing the certainty of summer precipitation estimate.

\section{Acknowledgment}

This work was financially supported by the National Science Foundation of China (No.41205025 and No.41175092), and the University graduate student's Scientific research innovation of Jiangsu Province China (No.CX10B_285Z) and Colleges and Universities naturnal science research project of Jiangsu China (No.12KJB1700127).

\section{References}

[1] AMITAI, E., X. Llort, and D. Sempere-Torre. (2009)

[2] Gourley, J. J., Y. Hong, Z. L. Flamig, L. Li, and J. Wang: Journal of Applied Meteorology and Climatology. (2010)

[3] Hirpa, F. A., M. Gebremichael, and T. Hopson: Journal of Applied Meteorology and Climatology. (2010)

[4] Hossain, F., and G. J. Huffman: Journal of Hydrometeorology. (2008)

[5] Huffman, G. J., R. F. Adler, E. Stocker, D. T. Bolvin, and E. J. Nelkin: Combined Preprints CD-ROM.(2003)

[6] Huffman, G. J., and Coauthors: Journal of Hydrometeorology. (2001)

[7] Morrissey, M. L., J. A. Maliekal, J. S. Greene, and J. Wang: Water Resources Research. (1995)

[8] Scofield, R. A., and R. J. Kuligowski: Weather and Forecasting.(2003) 
Advances in Environmental Technologies

10.4028/www.scientific.net/AMR.726-731

Evaluation of TRMM Summer Precipitation Over Huai-River Basin In China

10.4028/www.scientific.net/AMR.726-731.3401 